\title{
Cirugía herniaria electiva en pacientes portadores de tratamiento anticoagulante
}

\author{
Jaime Rappoport S. ${ }^{1}$, Nicole Cuneo B. ${ }^{1}$, Carlos Domínguez C. ${ }^{1}$, \\ Camila Sotomayor L. ${ }^{1}$, Carlos Jáuregui L. ${ }^{1}$ y Juan Jorge Silva S. ${ }^{1}$
}

\section{Elective hernia surgery in patients with anticoagulant treatment}

Introduction: Various pathologies require oral anticoagulant treatment (TACO). Some of these patients present pathologies of surgical resolution. The perioperative management of these patients is variable depending on the center. Aim: To evaluate the morbidity and mortality of patients attended with hernia pathology and TACO, assisted in our hospital. Materials and Method: Prospective, descriptive study of 37 patients submmited to hernia surgery in TACO between 2008-2016. The data was obtained from the computer database of the Hernia Team, with a minimum follow-up of 1 month. Clinical, surgical characteristics and postoperative morbidity and mortality were evaluated. The treatment overlap from TACO to Low Molecular Weight Heparin (LMWH) in therapeutic doses, was initiated three days before surgery. LMWH was suspended 24 hours prior to surgery, and reinitiated 12 to 24 hours post operation. 48 to 72 hours TACO was resumed. The data was analyzed with Stata v14. Results: Twenty-six patients were men, the mean age was 67.3 years. $48.7 \%$ had atrial fibrillation. $100 \%$ consumed acenocoumarol as TACO. The mean time for resuming TACO after surgery was 1.4 days. The average INR at the time of discharge was 2.04 . Two patients were discharged with dalteparin. One patient $(2.7 \%)$ presented pain in the immediate postoperative period and one showed ecchymosis of the surgical site (2.7\%). Conclusions: The work protocol used, proved to be safe, with minimal postoperative morbidity.

Key words: hernia; oral anticoagulant theraphy; inguinal; incisional; surgical treatment.

\section{Resumen}

Introducción: Diversas patologías requieren de tratamiento anticoagulante oral (TACO). Algunos de estos pacientes requieren resolución quirúrgica. El manejo perioperatorio de estos pacientes es variable dependiendo del centro. Objetivos: Evaluar la morbilidad y mortalidad del protocolo de manejo de patología herniaria en TACO, atendidos en nuestro hospital. Material y Métodos: Estudio descriptivo prospectivo de 37 pacientes sometidos a cirugía herniaria en TACO entre 2008-2016. Los datos fueron obtenidos de la base de datos computacional del Equipo de Hernias, con un seguimiento mínimo de 1 mes. Se evaluaron las características clínicas, quirúrgicas y la morbimortalidad postoperatoria. El traslape consistió en hospitalizar al paciente tres días previos a la cirugía, suspendiéndose el TACO e iniciando heparina de bajo peso molecular (HBPM) en dosis terapéuticas, que se suspende $24 \mathrm{~h}$ previas a la cirugía. Se reinicia la HPBM a las 12 a $24 \mathrm{~h}$ postoperatorias, y se inicia el traslape a TACO a las 24-48 h. Los datos fueron analizados con Stata v14. Resultados: De los 37 pacientes estudiados, veintiséis pacientes fueron hombres (70,2\%), la media de edad fue de 67,3 años. El 48,7\% tenían fibrilación auricular. El 100\% consumía acenocumarol como TACO. La media en el inicio del traslape a la anticoagulación oral fue de 1,4 días. El promedio de INR al momento del alta fue de 2,04. Dos pacientes fueron dados de alta con dalteparina. Un paciente $(2,7 \%)$, presentó dolor en el postoperatorio inmediato y uno $(2,7 \%)$, equimosis del sitio quirúrgico. Conclusiones: El protocolo de trabajo utilizado, demostró ser seguro, con una mínima morbilidad postoperatoria.

Palabras clave: hernia; tratamiento anticoagulante oral; inguinal; incisional; tratamiento quirúrgico.
'Departamento de Cirugía Hospital Clínico de la Universidad de Chile. Santiago, Chile.

Recibido el 20 de diciembre de 2018 y aceptado para publicación el 30 de enero de 2019.

Correspondencia a: Dr. Jaime Rappoport S. rapaj@usa.net 


\section{Introducción}

El constante aumento de la esperanza de vida ha generado que los pacientes sometidos a resolución quirúrgica de patología herniaria sean de mayor edad, asociándose a más comorbilidades ${ }^{1}$. En este último punto, destaca la presencia de fibrilación auricular, enfermedad tromboembólica o reemplazo valvular, donde hay indicación de utilización de terapia anticoagulante oral (TACO $)^{2}$. La suspensión del TACO lleva a aumento del riesgo tromboembólico, y aumentaría los costos asociados a la atención de salud lo que se relacionaría con mayor estadía hospitalaria debido al posible retraso del reinicio de la anticoagulación y al riesgo de desarrollar complicaciones en el postoperatorio ${ }^{3}$.

El propósito del presente estudio fue analizar la morbimortalidad de pacientes portadores de patología herniaria sometidos a cirugía electiva y en TACO, en el Hospital Clínico de la Universidad de Chile (HCUCH).

\section{Materiales y Método}

\section{Pacientes}

Se realizó un estudio descriptivo prospectivo, mediante la revisión del sistema computacional de registro del Equipo de Hernias de nuestro centro. Los criterios de inclusión fueron pacientes consumidores de anticoagulante oral (acenocumarol), que fueran sometidos a cirugía herniaria electiva. Se consideraron como criterios de exclusión: cirugía de urgencia, obesos con IMC mayor a 32, consumidores de nuevos anticoagulantes, pacientes que no siguieron el protocolo planteado por el grupo de trabajo.

Se incluyeron 37 pacientes sometidos a cirugía herniaria electiva, que necesitaron del traslape de anticoagulación preoperatorio, entre enero de 2008 y diciembre de 2016. La variable principal de este estudio, fue la morbilidad postoperatoria (hasta 30 días) definida como cualquier efecto adverso, en el postoperatorio, con especial énfasis en el sangrado postoperatorio y una eventual reoperación. Las variables secundarias estudiadas fueron: sexo, edad, comorbilidades, farmacoterapia previa, tipo de hernia, INR preoperatorio, días de hospitalización, cirugía, tiempo quirúrgico, instalación de drenajes, INR postoperatorio inmediato e INR al alta.

\section{Protocolo de tratamiento}

El traslape consistió en suspender el TACO 3 días previos a la cirugía. Se hospitaliza el paciente, se toma INR de ingreso. Si es mayor de 2,0 se in- dica vitamina $\mathrm{K} 10 \mathrm{mg} /$ día y control diario de INR. Se inicia el tratamiento anticoagulante con HBPM (5.000/7.500 ui de dalteparina, cada 12 h) hasta 24 $\mathrm{h}$ previas a la cirugía, momento en que se suspende el tratamiento. El día de la cirugía se toma un INR de control. Se consideró aceptable un INR menor a 1,5 para cirugía electiva.

Tras $12 \mathrm{~h}$ del postoperatorio se reinicia la HBPM en dosis anticoagulantes, y 24 a $48 \mathrm{~h}$ del postoperatorio se inicia el traslape a TACO.

Todos los pacientes firmaron un consentimiento informado para cirugía de hernia.

\section{Estadística}

Análisis descriptivo de los resultados mediante programa Stata ${ }^{\circledR}$ v14.

\section{Resultados}

\section{Datos demográficos}

Este estudio incluyó a 37 pacientes (11 mujeres, 26 hombres), con una edad promedio de 67,3 años (rango 43-84). La comorbilidad asociada a la anticoagulación, más frecuente fue la fibrilación auricular en $18(48,7 \%)$ pacientes, seguida por recambio de válvula cardíaca en $9(24,3 \%)$ pacientes y antecedente de trombosis venosa en $4(10,8 \%)$ pacientes. Todos usuarios de acenocumarol como anticoagulante.

\section{Clínica}

La localización más frecuente de la hernia fue inguinal en 28 pacientes $(75,7 \%)$ (Tabla 1$)$. Todos los pacientes contaban con ecotomografía de pared abdominal. El INR de ingreso promedio fue de 2,04 (rango 1,05-4,6). Todos los pacientes se hospitalizaron 3 días previos al procedimiento quirúrgico para iniciar el traslape. A dos pacientes $(5,4 \%)$, se le administró vitamina $\mathrm{K}$ al momento del ingreso. El INR en el preoperatorio inmediato, fue en promedio de 1,24 (rango 1,05-1,52).

\section{Resolución quirúrgica}

Todos los procedimientos se realizaron en forma electiva. El tiempo quirúrgico promedio fue de

Tabla 1. Localización de la hernia

\begin{tabular}{|lr|}
\hline Localización & n $(\mathbf{\%})$ \\
\hline Inguinal & $29(78,4)$ \\
\hline Umbilical & $4(10,9)$ \\
\hline Incisional & $4(10,9)$ \\
\hline
\end{tabular}


95 min (rango 40-330). La cirugía más frecuente fue la hernioplastía inguinal en 28 pacientes $(75,7 \%)$ (Tabla 2). En treinta y cinco de los pacientes $(94,6 \%)$, se realizó por vía abierta y dos pacientes $(5,4 \%)$, por vía laparoscópica. En relación a las complicaciones en el postoperatorio inmediato, 2 $(5,4 \%)$ pacientes presentaron dolor, y ninguno presentó complicaciones hemorrágicas o trombóticas. La media en el inicio del traslape postoperatorio a la anticoagulación oral fue de 1,4 días (1 a 14 rango). La media de INR al momento del reinicio del traslape, en el postoperatorio, fue de 1,23 (rango 0,95-1,8) y la media de INR al momento del alta fue de 2,04 (rango 1,08-4,6). El tiempo promedio de hospitalización fue de 6,11 días (rango 2-14). Dos pacientes fueron dados de alta con dalteparina. Dos pacientes presentaron morbilidad perioperatoria (Tabla 3).

La presente serie no registró mortalidad operatoria.

\section{Discusión}

Los pacientes sometidos a TACO, deben ser sometidos a un proceso de interrupción transitoria de esta terapia, para poder realizar una cirugía electiva, con bajo riesgo de complicaciones hemorrágicas. Esto se denomina traslape y generalmente se utiliza heparina de bajo peso molecular en este periodo, creando una ventana sin anticoagulación para efectuar la cirugía 4 .

Algunos autores, han propiciado la mantención de la terapia de TACO, pero esto se asocia a un aumento de las complicaciones hemorrágicas en el postoperatorio 5 .

Por otra parte la suspensión de la terapia de TACO, puede presentar el llamado efecto de rebote con hipercoagubilidad, aumentado el riesgo de desarrollar una nueva enfermedad tromboembólica ${ }^{6}$. Este riesgo se relaciona con la patología de base y el tiempo desde el evento inicial que gatilló el inicio de la anticoagulación. Es así, como la interrupción del TACO en pacientes con trombosis venosa profunda (TVP) o embolía pulmonar (EP), tienen un riesgo anual de recurrencia cercano al 5\%, pero este riesgo aumenta a un $40 \%$ dentro del primer mes posterior a la TVP/EP 2,6 . Dunn et al., realizaron una revisión sistemática sobre el manejo perioperatorio de los pacientes con TACO, evidenciándose la presencia de 29 eventos tromboembólicos en 1.868 pacientes $(1,6 \%)$. De estos 29 eventos, uno se dio en pacientes que continuaron con TACO $(0,4 \%), 6$ en aquellos que suspendieron TACO sin traslape $(0,6 \%), 0$ en aquellos con traslape a heparina intravenosa $(0 \%)$,
Tabla 2. Descripción del tipo de cirugía realizada

\begin{tabular}{|lr|}
\hline Tipo cirugía & n $(\%)$ \\
\hline Hernioplastía inguinal & $28(75,7)$ \\
\hline Hernioplastia umbilical & $4(10,9)$ \\
Hernioplastía incisional & $4(10,9)$ \\
Hernioplastía femoral. & $1(2,7)$ \\
\hline
\end{tabular}

Tabla 3. Complicaciones perioperatorias según Clavien Dindo

\begin{tabular}{|c|c|}
\hline Clavien Dindo & n (\%) \\
\hline Tipo I & $2(5,4)$ \\
\hline Dolor & $1(2,7)$ \\
\hline Equimosis & $1(2,7)$ \\
\hline
\end{tabular}

uno en aquellos que traslaparon a heparina de bajo peso molecular (HBPM) $(0,6 \%)$ y 21 en aquellos con esquema poco claro $(8,0 \%)^{4}$. Estos hallazgos recalcan la necesidad de contar con un esquema preciso de manejo en estos pacientes.

Por otra parte, el riesgo de desarrollar complicaciones hemorrágicas en el postoperatorio, debe ser evaluado al momento de definir qué se hará con los pacientes que se encuentran en TACO. Dunn et al., plantean que en base a los datos disponibles al momento de su trabajo, la probabilidad de eventos hemorrágicos mayores al segundo día postoperatorio sería de entre un 2 y $4 \%$ para cirugía mayor y 0 a $2 \%$ para procedimientos invasivos ${ }^{4}$.

Los pacientes sometidos a TACO, representan un subconjunto de pacientes, con patologías de base severas, y se ha asociado a mayor riesgo de complicaciones hemorragíparas o trombóticas, en el postoperatorio ${ }^{1-3,7}$.

El manejo perioperatorio, varía de un centro a otro, pero Dunn enfatiza que los grupos que utilizan un esquema predefinido de manejo, tienen resultados superiores a aquellos grupos en que no existe un protocolo de manejo, y cada paciente es manejado de forma diversa ${ }^{4}$.

Los diversos estudios publicados, son muy heterogéneos, en cuanto al tipo de patología, la indicación de TACO, el tipo de fármaco utilizado, series de casos, estudios cooperativos o artículos de revisión, por lo cual es difícil extraer conclusiones precisas acerca del manejo perioperatorio en estos pacientes (Tabla 4) $)^{1,2,4,5,7-11}$. Son diferentes los ries- 
Tabla 4. Revisión de estudios que evalúan la anticoagulación perioperatoria.

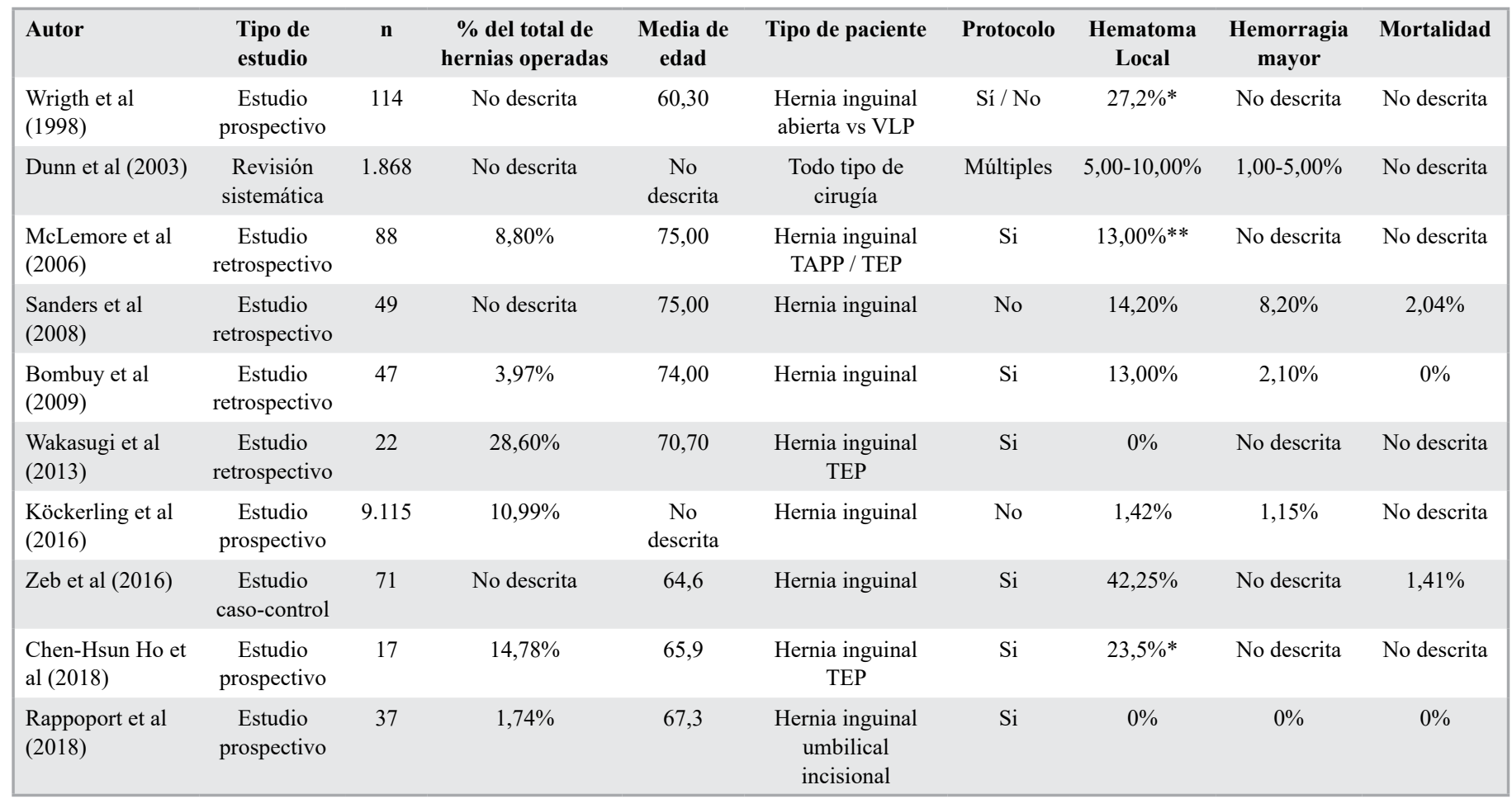

*Incluye en la estadística seromas. **En 2/15 pacientes que descontinuaron TACO con terapia puente.

gos de complicaciones en diversas patologías como cirugía ocular, dental, cutánea, traumatológica, cardiaca, patología herniaria, etc ${ }^{4}$.

En la literatura nacional encontramos dos publicaciones del tipo de artículos de revisión y recomendaciones de manejo, sin casuísticas ${ }^{12,13}$. Hasta nuestro conocimiento, este trabajo sería la primera comunicación en el manejo perioperatorio de pacientes portadores sólo de patología herniaria sometidos a terapia de TACO, con un protocolo de trabajo y casuística propia, a nivel nacional.

\section{Conclusiones}

Los resultados del presente estudio, sugieren que el protocolo de trabajo utilizado, si bien alarga la hospitalización de estos pacientes, demostró ser efectivo y seguro, en términos de minimizar las complicaciones asociadas a sangrado postoperatorio o complicaciones trombóticas.

Un mayor número de casos y la eventual adopción de otros grupos de trabajo del presente esquema, permitirán evaluar la real utilidad del protocolo de manejo perioperatorio de pacientes sometidos a cirugía electiva en TACO.

\section{Responsabilidades éticas}

Protección de personas y animales. Los autores declaran que para esta investigación no se han realizado experimentos en seres humanos ni en animales.

Confidencialidad de los datos. Los autores declaran que en este artículo no aparecen datos de pacientes.

Conflictos de interés: no hay. 


\section{Bibliografía}

1. Wakasugi M, Akamatsu H, Yoshidome K, Tori M, Ueshima S, Omori T, et al. Totally extraperitoneal inguinal hernia repair in patients on antithrombotic therapy: a retrospective analysis. Surg Today 2013;43:942-5.

2. Sanders DL, Shahid MK, Ahlijah B, Raitt JE, Kingsnorth AN. Inguinal hernia repair in the anticoagulated patient: a retrospective analysis. Hernia 2008;12:589-92; discussion 667-8.

3. Watts SA, Gibbs NM. Outpatient management of the chronically anticoagulated patient for elective surgery. Anaesth Intensive Care 2003;31:145-54.

4. Dunn AS, Turpie AG. Perioperative management of patients receiving oral anticoagulants: a systematic review. Arch Intern Med. 2003;163:901-8.

5. Ho CH, Wu CC, Wu CC, Tsai YC.
Laparoscopic total extraperitoneal inguinal hernia repair is safe and feasible in patients with continuation of antithrombotics. J Minim Access Surg. 2018;128:18-24.

6. Kearon C, Hirsh J. Management of anticoagulation before and after elective surgery. N Engl J Med. 1997;336:1506-11.

7. Kockerling F, Roessing C, Adolf D, Schug-Pass C, Jacob D. Has endoscopic (TEP, TAPP) or open inguinal hernia repair a higher risk of bleeding in patients with coagulopathy or antithrombotic therapy? Data from the Herniamed Registry. Surg Endosc. 2016;30:2073-81.

8. Bombuy E, Mans E, Hugue A, Plensa E, Rodríguez L, Prats M, et al. Elective inguinal hernioplasty in patients on chronic anticoagulation therapy. Management and outcome. Cir Esp. 2009;86:38-42.

9. McLemore EC, Harold KL, Cha SS,
Johnson DJ, Fowl RJ. The safety of open inguinal herniorraphy in patients on chronic warfarin therapy. Am J Surg. 2006;192:860-4.

10. Wright DM, O'Dwyer PJ, Paterson CR. Influence of injection site for low-dose heparin on wound complication rates after inguinal hernia repair. Ann R Coll Surg Engl. 1998;80:58-60.

11. Zeb MH, Pandian TK, El Khatib MM, Naik ND, Chandra A, Morris DS, et al. Risk factors for postoperative hematoma after inguinal hernia repair: an update. $\mathrm{J}$ Surg Res. 2016;205:33-7.

12. AM. E. Tromboprofilaxis en el paciente quirúrgico. Rev Chil Anestesia 2008;37:920.

13. Nazar C, Coloma R, Contreras JI, Molinari I, Miranda P, Fuentes R. Manejo perioperatorio de pacientes con tratamiento anticoagulante crónico. Rev Chil Cir. 2017;70:84-91. 